\title{
Atopic eczema
}

\author{
Author: Sara J Brown ${ }^{\mathrm{A}}$
}

Atopic eczema is an itchy inflammatory skin disease with a chronic relapsing-remitting course; it has increased in prevalence in recent decades and now affects up to $25 \%$ of school-aged children in the developed world and up to $10 \%$ of adults. Recent advances in understanding the aetiology of eczema have focused interest on skin barrier dysfunction as a common precursor and pathological feature. In addition, genetically determined skin barrier dysfunction (associated with mutations in the gene encoding filaggrin) is known to predispose to multiple systemic atopic diseases. Firstline treatments for atopic eczema focus on maintaining and repairing the skin barrier (emollients) and reducing inflammation (topical steroids); allergen and irritant avoidance are also important to achieve disease control. Second and third-line treatments include topical calcineurin inhibitors, ultraviolet light and systemic immunosuppressant therapies of which only ciclosporin is licenced for the treatment of atopic eczema in adults. Novel biological therapies are in phase II-III clinical trials.

\section{Importance of eczema and its comorbidities}

Atopic eczema (synonymous with atopic dermatitis and eczema ${ }^{1}$ ) is an itchy inflammatory skin disease which follows a chronic relapsing and remitting course. Moderate-severe eczema (Fig 1) has a significant impact on the quality-of-life of affected patients and their close family because of itching, scratching and sleep deprivation; the impairment in quality-oflife is of a similar magnitude to epilepsy or type- 1 diabetes in childhood. ${ }^{2}$ In approximately $40 \%$ of cases eczema persists into adulthood (Fig 2), having an impact on work productivity and social functioning. ${ }^{3}$

Eczema is a complex trait, resulting from the interaction of multiple genetic and multiple environmental factors. Familial clustering and twin studies demonstrate that the disease is highly heritable, emphasising the strong role of genetic predisposition, ${ }^{4}$ while the rapid rise in prevalence of eczema in the developed world emphasises the importance of environmental factors.

Author: ${ }^{\text {A }}$ rofessor of molecular and genetic dermatology, Wellcome Trust senior research fellow in clinical science and honorary consultant dermatologist, Skin Research Group, Ninewells Hospital and Medical School, Dundee, UK

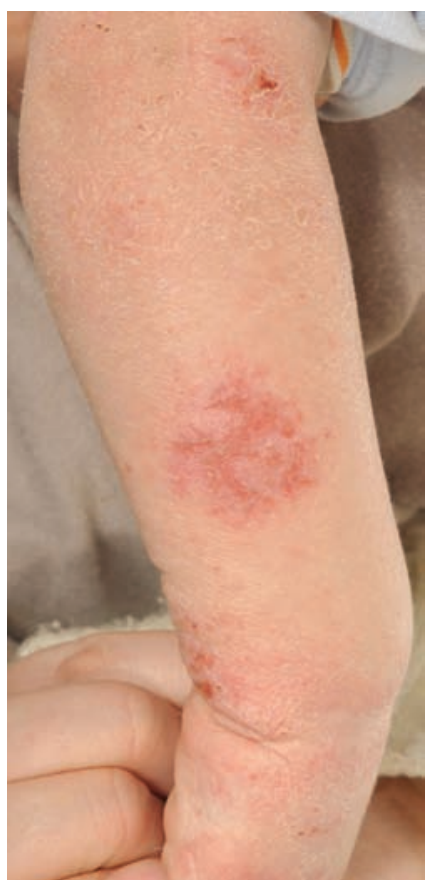

Fig 1. Child with moderate-severe atopic eczema. The child's upper limb shows multiple areas of eczematous inflammation in flexural and nonflexural sites, on a background of generalised ichthyosis vulgaris (common dry, scaly skin).

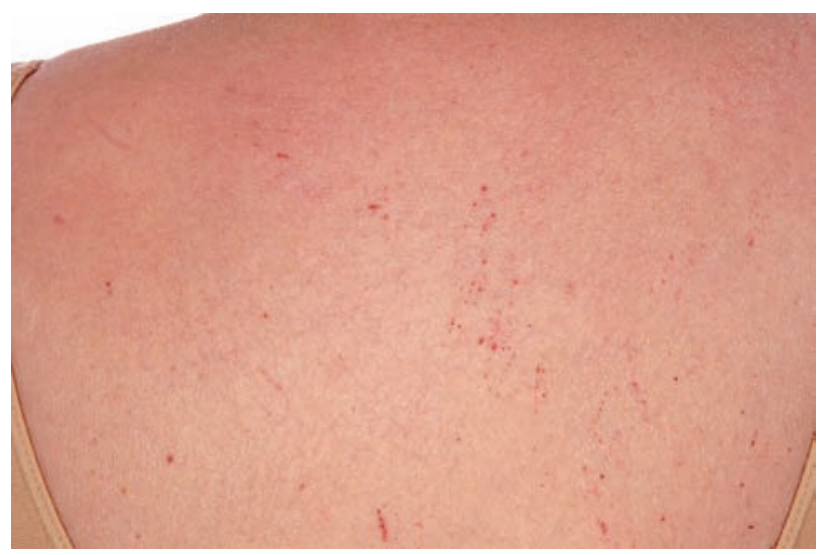

Fig 2. Adult severe atopic eczema. The skin on this adult's upper back shows widespread erythema and scaling, indicative of active eczema, with multiple excoriations. 
Up to $40 \%$ of eczema cases have atopic comorbidities including asthma, allergic rhinitis and type-1 hypersensitivity to food, which may be initiated by transcutaneous sensitisation. ${ }^{5}$ These comorbidities add substantially to the disease burden. The chronic sleep disturbance resulting from nocturnal pruritus in eczema can lead to neurocognitive impairment. An association with neurodevelopmental disorders, including attention deficit hyperactivity disorder, has also been observed from epidemiological studies. ${ }^{6}$

\section{Diagnostic criteria}

Eczema is diagnosed on the basis of clinical features: illdefined, erythematous, scaly and pruritic lesions with a clinical history characterised by childhood onset, flexural inflammation (although non-flexural sites are usually involved in early infancy), a background of generalised skin dryness (xerosis and/or ichthyosis vulgaris) and associated atopic disease in the patient and/or first-degree relatives. ${ }^{3}$ Eczema can occur at any body site but in atopic patients the skin as a whole organ shows impairment in barrier function and a predisposition to inflammation, even in clinically uninflamed sites. Barrier dysfunction also manifests as skin dryness, in part because of increased water loss, and susceptibility to viral and bacterial infections.

\section{Principles of managing atopic eczema}

The main principles of eczema management are allergen and irritant avoidance, emollient use and topical or systemic antiinflammatory treatment (Box 1). The most common allergens leading to sensitisation in childhood are house-dust mite,

\section{Key points}

Atopic eczema (synonymous with atopic dermatitis and eczema) is an itchy inflammatory skin disease with significant impact on quality of life.

Skin barrier dysfunction in the atopic patient facilitates allergen and irritant penetration leading to skin and systemic inflammation, including eczema, atopic asthma, allergic rhinitis and peanut allergy.

The diagnosis of atopic eczema is based on clinical history and examination.

Treatments for eczema include allergen and irritant avoidance, emollients, topical steroids, calcineurin inhibitors, ultraviolet radiation and systemic anti-inflammatory agents, plus the treatment of secondary infection when present.

Urgent and emergency presentations of eczema are often associated with secondary infections including $S$ aureus and herpes simplex virus (as eczema herpeticum).

KEYWORDS: Atopic, barrier, dermatitis, eczema and filaggrin

\section{Box 1. Principles of treatment in atopic eczema.}

Avoid known allergens and common skin irritants (eg soap and detergents).

Use emollients to wash the skin with and to apply directly onto skin.

Apply topical corticosteroid ointment (or cream when ointment is not tolerated) of appropriate strength, once daily, aiming to 'switch off' the eczema. Then reduce potency and/or frequency of steroid application aiming to maintain remission.

Treat secondary infection, bacterial and/or viral, when this is present.

Topical calcineurin inhibitors can be used as second-line treatment for sites at high risk of corticosteroid-induced skin atrophy, eg facial skin. They may also be applied once-twice weekly to help maintain remission.

Other second- and third-line treatments include ultraviolet $\mathrm{B}$, ciclosporin, methotrexate (unlicensed indication) and azathioprine (unlicensed indication).

Biologic agents for atopic eczema are currently in clinical trials.

grass pollen, milk, egg and peanut. Allergens inducing a type-1 hypersensitivity reaction should clearly be avoided and there is some evidence that reducing dust-mite exposure in the subset of sensitised patients improves eczema control. ${ }^{7}$ The most common irritants encountered in everyday life are soap and detergents; clinical experience demonstrates the utility of soap substitutes (emollients) when bathing, but clinical trial data for this relatively inexpensive intervention are currently lacking. ${ }^{8}$ Topical corticosteroids should be applied to areas of active eczema at a potency aimed to induce remission, and a steroid of lower potency or less frequent application used to maintain remission. ${ }^{3}$ Topical steroids should not be applied at the same time as emollients and some specialists recommend application after bathing to optimise penetration through the stratum corneum.

Second- and third-line treatments include topical calcineurin inhibitors (pimecrolimus and tacrolimus), ultraviolet radiation (principally narrow-band UVB phototherapy), oral corticosteroids and the systemic immunosuppressants, ciclosporin, azathioprine, methotrexate and mycophenolate mofetil. However, ciclosporin is the only systemic immunosuppressant currently licensed in the UK for adults; systemic treatment in children is currently off licence but ciclosporin, azathioprine and methotrexate are each used by dermatology specialists in the UK and internationally. ${ }^{9,10}$

Secondary infection with bacteria (most frequently Staphylococcus aureus) is very commonly associated with eczema exacerbations and should be treated concomitantly. Eczema herpeticum is an infection of herpes simplex virus within areas of skin affected by eczema; it may present as a medical emergency (see below).

A recent analysis of systematic reviews has concluded that there is a paucity of evidence for complementary eczema treatments and non-pharmacological interventions such as specialist clothing; further well-designed clinical trials are needed. ${ }^{11}$ 


\section{Atopic eczema in acute medicine}

Patients with atopic eczema may present in the acute setting with exacerbations of their comorbidities including asthma or anaphylaxis. Acute exacerbations of eczema (so-called 'flareups') are often associated with $S$ aureus infection. Whether this is cause or effect is uncertain, ${ }^{12}$ but eczema control requires treatment of the infection (usually with a short course of oral flucloxacillin) in parallel with treatment of the atopic inflammation. ${ }^{3}$

Herpes simplex virus produces a severe and potentially life-threatening infection of eczematous skin, described as eczema herpeticum. This condition presents clinically with eczema flare, pain, systemic upset and characteristic monomorphic vesicular and punched-out lesions. It warrants urgent referral to a dermatology specialist for assessment and prompt intervention with systemic antiviral therapy (acyclovir or valaciclovir), which may need to be given parentally, with anti-staphylococcal therapy if indicated. Periorbital involvement necessitates ophthalmological review because of the risk of corneal ulceration. Supportive treatment, including hospital admission, intravenous fluid replacement and careful monitoring, may be required in extensive eczema herpeticum; particularly if the patient is immunosuppressed since mortality may be as high as $10 \%$ in this group of patients.

Widespread dermatitis can contribute to the morbidity and mortality of multiorgan failure, therefore when present it should be treated actively. The differential diagnosis in this context includes atopic dermatitis, irritant or allergic contact dermatitis, stasis dermatitis and scabies infestation.

\section{Advances in understanding the aetiology and pathogenesis of eczema}

The discovery in 2006 of loss-of-function mutations in FLG, the gene encoding a skin barrier protein filaggrin ${ }^{13}$ and their role in eczema predisposition ${ }^{14}$ led to a paradigm shift in understanding the pathogenesis of atopic eczema. ${ }^{5}$ This basic scientific discovery has illustrated the underlying role of skin barrier dysfunction in the development of skin and systemic atopic diseases. ${ }^{4}$ A genetically determined filaggrin deficiency in the skin significantly increases risk of each disease in the socalled 'atopic march', including eczema, atopic asthma, allergic rhinitis and peanut allergy. ${ }^{4,15}$ Skin barrier dysfunction may be quantified in part by trans-epidermal water loss (TEWL) and increased TEWL is present as a precursor to eczema in early infancy. ${ }^{16}$ Building on this observation, recent clinical trials have produced evidence to suggest that intensive emollient use from soon after birth, aimed to enhance the skin barrier, reduces the incidence of eczema by $\sim 50 \%$ in high-risk infants (defined as those with a first-degree relative having atopic disease).${ }^{17}$ However, the authors emphasise that larger trials are required before this approach can be recommended for routine clinical practice.

Despite these major advances in understanding eczema pathogenesis, there remain considerable gaps in knowledge. More than 30 genetic risk loci have now been identified by genome-wide association studies in atopic eczema but the majority of these are in intergenic regions with unknown function. Eczema currently lags behind psoriasis and other systemic inflammatory diseases in the availability of biological therapies. However, the results of a phase-II clinical trial investigating dupilumab (a human monoclonal antibody against interleukin-4 and interleukin-13) in adults with moderate-severe atopic eczema are promising: $85 \%$ of patients in the dupilumab group, as compared with $35 \%$ of those in the placebo group, had a 50\% reduction in the eczema severity score $(\mathrm{p}<0.001)$ after 12 weeks of monotherapy. ${ }^{18} \mathrm{~A}$ phase-IIb trial has shown a dose-dependent improvement in adults with moderate-to-severe atopic eczema; ${ }^{19}$ there were no significant safety concerns reported, although there was an increased incidence of cutaneous herpes virus infections in the dupilumab-treated group, a risk which will require further assessment.

Another area of intensive research interest is the complex interaction of skin and gut microbiome with cutaneous inflammation. ${ }^{12}$ These studies which have been made possible by modern sequencing technologies to circumvent the need for selective bacterial cultures. ${ }^{20}$ The approach has illustrated a reduction in microbial diversity in active eczema in addition to the well-established overgrowth of $S$ aureus. ${ }^{12}$

\section{Conclusions and future prospects}

In conclusion, atopic eczema represents a complex and challenging disorder for both patient and physician. Simple interventions, including the avoidance of allergens and irritants, combined with emollient use and topical corticosteroids, provide the mainstay of treatment and are effective in the majority of cases. A subset of severe recalcitrant disease necessitates systemic immunosuppression, but more targeted and effective therapies are urgently required. Recent advances in understanding both genetic and environmental factors in eczema pathogenesis offer the opportunity for significant progress in this regard. The diverse range of phenotypes observed within the clinical diagnosis of 'eczema' illustrates the need for a personalised medicine approach, to target novel therapies to subsets of patients whose disease is driven by specific pathways. The emerging knowledge of tissue microbial diversity and its role in modulating inflammatory disease also offers opportunity for therapeutic manipulation of microbial communities in the future. ${ }^{20}$

\section{Acknowledgements}

I am grateful to Professor Nick Reynolds and Dr Carsten Flohr for their critical reading and comments on this manuscript. Clinical images are reproduced by the University of Dundee Computing and Media Services, Ninewells Hospital and Medical School, with patient/parental consent.

\section{Funding}

SJB is funded by a Wellcome Trust Senior Research Fellowship in Clinical Science (ref 106865/Z/15/Z).

\section{References}

1 Johansson SG, Bieber T, Dahl R et al. Revised nomenclature for allergy for global use: Report of the Nomenclature Review Committee of the World Allergy Organization, October 2003. J Allergy Clin Immunol 2004;113:832-6. 
2 Beattie P, Lewis-Jones M. A comparative study of impairment of quality of life in children with skin disease and children with other chronic childhood diseases. Br J Dermatol 2006;155:145-51.

3 Williams HC. Clinical practice. Atopic dermatitis. N Engl J Med 2005;352:2314-24.

4 Brown SJ, McLean WH. One remarkable molecule: filaggrin. J Invest Dermatol 2012;132:751-62.

5 Irvine AD, McLean WH, Leung DY. Filaggrin mutations associated with skin and allergic diseases. N Engl J Med 2011;365:1315-27.

6 Deckert S, Kopkow C, Schmitt J. Nonallergic comorbidities of atopic eczema: an overview of systematic reviews. Allergy 2014; 69:37-45.

7 Nankervis H, Pynn EV, Boyle RJ et al. House dust mite reduction and avoidance measures for treating eczema. Cochrane Database Syst Rev 2015;1:CD008426.

8 No authors listed. Bath emollients for atopic eczema: why use them? Drug Ther Bull 2007;45:73-5.

9 Proudfoot LE, Powell AM, Ayis S et al. The European TREatment of severe Atopic eczema in children Taskforce (TREAT) survey. $\mathrm{Br} \mathrm{J}$ Dermatol 2013;169:901-9.

10 Flohr C, Irvine AD. Systemic therapies for severe atopic dermatitis in children and adults. J Allergy Clin Immunol 2013;132:774-e6.

11 Madhok V, Futamura M, Thomas KS, Barbarot S. What's new in atopic eczema? An analysis of systematic reviews published in 2012 and 2013. Part 2. Treatment and prevention. Clin Exp Dermatol 2015; 40:349-54;quiz 54-5.

12 Kong HH, Oh J, Deming C, Conlan S et al. Temporal shifts in the skin microbiome associated with disease flares and treatment in children with atopic dermatitis. Genome Res 2012;22:850-9.

13 Smith FJ, Irvine AD, Terron-Kwiatkowski A et al. Loss-of-function mutations in the gene encoding filaggrin cause ichthyosis vulgaris. Nat Genet 2006;38:337-42.
14 Palmer CN, Irvine AD, Terron-Kwiatkowski A et al. Common lossof-function variants of the epidermal barrier protein filaggrin are a major predisposing factor for atopic dermatitis. Nature Genet 2006;38:441-6.

15 Rodriguez E, Baurecht H, Herberich E et al. Meta-analysis of filaggrin polymorphisms in eczema and asthma: robust risk factors in atopic disease. J Allergy Clin Immunol 2009;123:1361-70.e7.

16 Kelleher M, Dunn-Galvin A, Hourihane JO et al. Skin barrier dysfunction measured by transepidermal water loss at 2 days and 2 months predates and predicts atopic dermatitis at 1 year. J Allergy Clin Immunol 2015;135:930-5.e1.

17 Simpson EL, Chalmers JR, Hanifin JM et al. Emollient enhancement of the skin barrier from birth offers effective atopic dermatitis prevention. J Allergy Clin Immunol 2014;134:818-23.

18 Beck LA, Thaci D, Hamilton JD et al. Dupilumab treatment in adults with moderate-to-severe atopic dermatitis. $N$ Engl J Med 2014;371:130-9.

19 Thaci D, Simpson EL, Beck LA et al. Efficacy and safety of dupilumab in adults with moderate-to-severe atopic dermatitis inadequately controlled by topical treatments: a randomised, placebo-controlled, dose-ranging phase $2 \mathrm{~b}$ trial. Lancet 2015, epub ahead of print.

20 Grice EA. The intersection of microbiome and host at the skin interface: genomic- and metagenomic-based insights. Genome Res 2015;25:1514-20.

Address for correspondence: Prof S] Brown, Skin Research Group, School of Medicine, Ninewells Hospital and Medical School, Jacqui Wood Centre Level 7, James Arrott Drive, University of Dundee, Dundee DD1 9SY, UK.

Email: s.j.brown@dundee.ac.uk 